\title{
Beyond $\mathrm{KCH}$ selection and options in acute liver failure
}

\author{
William Bernal ${ }^{1} \cdot$ Roger Williams ${ }^{2,3}$
}

Received: 24 February 2018 / Accepted: 24 April 2018/Published online: 1 June 2018

(C) The Author(s) 2018

\begin{abstract}
The King's prognostic criteria for patients with acute liver failure (ALF) introduced in 1989 have been used worldwide. This distinguished for the first time cases with 'hyper-acute' course (characteristically paracetamol overdose) where there is a better chance of recovery with medical supportive care alone from those etiologies with a less acute course and paradoxically lower chances of 'spontaneous' recovery. Ongoing use showed the limited sensitivity of the criteria to constitute a significant practical limitation. Subsequent models including the MELD score and composite ones with markers of necrosis, an apoptotic liver cell death, proposed to improve sensitivity did not have the required high specificity. Two recent models utilizing new availability of web- and app-based computing delivering outcome predication through sophisticated algorithms are described. The first is a dynamic model described for paracetamol-induced ALF based upon admission findings and sequential variables over the first 2 days. The new model of the US Acute Liver Failure group was devised to cover all etiologies of ALF for predicting 'transplant-free' survival and accurately predicated spontaneous survival in two-thirds of cases. Improved survival results with medical management, particularly in hyper-acute cases, now approach those obtained with successful liver transplant and have raised the question of transplant benefit. Also considered in the review are new non-transplant approaches to treatment including the use of plasma exchange and based on successful results in acute-on-chronic liver failure, agents to modulate and improve hepatic regeneration.
\end{abstract}

Keywords Acute liver failure $\cdot$ King's criteria $\cdot$ Dynamic model $\cdot$ ALFSG $\cdot$ Plasma exchange $\cdot$ Liver transplant

\section{Introduction and historical context}

Difficulties in determining prognosis in patients with acute liver failure (ALF) from paracetamol hepatotoxicity have been apparent for many years. Initial issues related to the difficulties in identifying those patients with paracetamol intoxication who would benefit from antidotal therapy [1]. The need for accurate predictive tests of patient outcome became even more apparent when the life-saving procedure of liver transplant for patients with ALF was introduced in the early 1980. Concerns were raised that some patients

Roger Williams

r.williams@ researchinliver.org.uk

1 The Institute of Liver Studies, Cheyne Wing, King's College Hospital, Denmark Hill, London SE5 9RS, UK

2 The Institute of Hepatology London and Foundation for Liver Research, 111 Coldharbour Lane, London SE5 9NT, UK

3 Faculty of Life Sciences \& Medicine, King's College, London, UK would be transplanted who would otherwise have recovered with medical management alone; variously termed 'transplant-free' or 'spontaneous' survival.

The description of the King's College Criteria (KCC) in 1989 represented a major step forward in identifying candidates for liver transplantation (Text Box 1) [2]. It stressed the importance of etiology and mode of presentation in the outcome of ALF, recognizing the chances of recovery with medical supportive care alone with rapidly evolving paracetamol hepatotoxicity as being much higher than with other etiologies of ALF, particularly those with illness of more gradual onset. Derived and validated in a cohort of 763 patients managed before the introduction of transplantation for ALF, the high specificity of the KCC meant that relatively few cases fulfilling criteria would be unnecessarily transplanted. However, their sensitivity was lower, indicating that a significant number of cases not fulfilling criteria would progress and die without earlier identification and consideration for possible liver transplantation. For the non-paracetamol cases, both sensitivity and specificity were high but within this overall group, 
certain etiologies with rapid illness onset—specifically ischemic hepatitis, acute fatty liver of pregnancy and hepatitis A-had similar survival rates with medical management alone to that of paracetamol-induced cases. It was in those cases with an indolent presentation and 'sub-acute' phenotype and often 'indeterminate' etiology that had the worst outcome without transplantation [3].

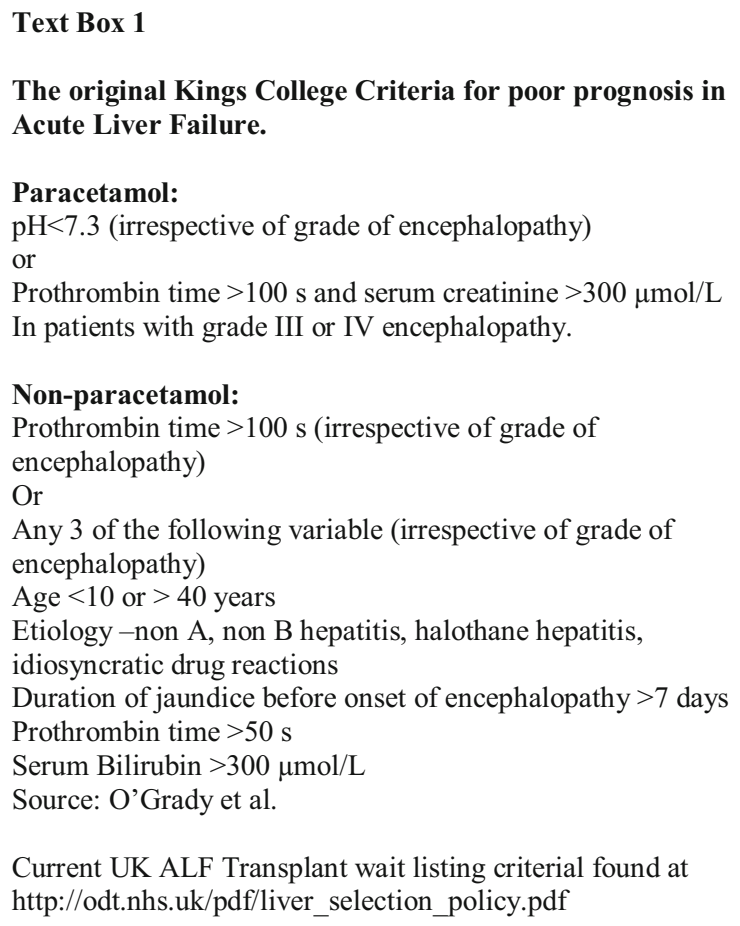

Decision making in relation to the use of transplantation in ALF became even more difficult as improvements in intensive liver care led to higher survival rates with medical management alone. In the King's College Hospital $(\mathrm{KCH})$ experience of over 3000 patients treated between 1973 and 2008, transplant-free survival had increased to $48 \%$, with a near fourfold increase in overall hospital survival rates-including cases transplanted from 16.7 to $62.2 \%$ [4].The increase in 'transplant-free' survival was most apparent in paracetamol cases and associated with a substantial fall in the occurrence of cerebral edema and intracranial hypertension, which in the past was often the cause of death in cases with a 'hyper-acute' presentation. An important contribution to this improvement is likely through control of circulating levels of ammonia-the principal neurotoxin in this setting-through interventions that include the earlier and more widespread use of continuous hemofiltration [5, 6]. In paracetamol-induced ALF, the narrowing in the gap between survival with medical management alone and survival with transplantation has raised doubts about the transplant benefit in such cases [7].
These changes in the spectrum of complications and improvement in survival have since been documented in other large historical series [8-10].

In this review, we will consider: first, the basis of prognostic assessment systems in ALF and the practical difficulties in their application, with focus upon the KCC as an example of a system in current use. Second, the improved results currently being obtained with liver transplantation in these very sick patients are described and third, how new approaches to treatment may impact upon future care and outcome.

\section{Improving prediction of outcome}

A number of different systems are in current use for the evaluation of prognosis in patients with ALF, with clinical experience of their application and operational performance reported for the KCC and criteria from France and Japan [2, 10-12]. Though details of the models used vary, they share common features (Table 1). All recognize the key importance of the development of encephalopathy as a marker of critically impaired liver function and retain it as a central criterion of poor prognosis. All also utilize laboratory measures of liver function to quantify severity of liver injury, most common measures of coagulation status. Patient Age is also recognized by all as being of prognostic importance-likely reflecting the compromise of physiologic reserve with increasing age- - key to surviving any critical illness, and a possible parallel impairment of hepatic regenerative ability. An important difference of the $\mathrm{KCC}$ from the other established criteria is the distinction between paracetamol and non-paracetamol etiologies, reflecting the high volume of paracetamol cases seen in the United Kingdom and differences seen in clinical course. Their clinical simplicity has, however, served them well over time, relying not on complex calculation or use of specialized laboratory tests but rather standard bedside

Table 1 Comparison of elements of prognostic scoring systems in current widespread use

\begin{tabular}{llll}
\hline Factor & \multicolumn{2}{l}{ Criteria } & \\
\cline { 2 - 4 } & Kings $^{\mathrm{b}}$ & Clichy $^{\mathrm{c}}$ & Japanese $^{\mathrm{d}}$ \\
\hline Age $^{\mathrm{a}}$ & + & + & + \\
Etiology $_{\text {Encephalopathy }}^{\mathrm{a}}$ & + & - & - \\
Bilirubin $_{\text {Coagulopathy }}^{\mathrm{a}}$ & + & + & + \\
\hline
\end{tabular}

+ Factor included in criteria. - Factor not included in criteria

${ }^{\mathrm{a}}$ Factor common to all criteria. References: ${ }^{\mathrm{b}}[2],{ }^{\mathrm{c}}[56],{ }^{\mathrm{d}}[57]$ 
assessment and readily available routine laboratory measures.

Published data illustrate their limitations in current clinical practice, particularly in relation to hyper-acute disease. Here the initial intensity of critical illness may be severe but paradoxically the potential for native liver regeneration is high $[4,9,11]$. In early case series of hyperacute patients, the dominant clinical issues were those of either a failure to identify all patients with a poor prognosis for consideration of transplantation-limited sensitivityin concert with that of rapid deterioration of those who fulfilled KCC and high risk of death on the transplant waitlist prior to grafts becoming available, late identification [13-15]. Efforts were, therefore, initially focussed on refining the $\mathrm{KCC}$ by improving criteria sensitivity enabling earlier identification of patients with a poor prognosis through integration of supplemental prognostic markers.

Initial studies explored the inclusion of arterial blood lactate measurements determined using point of care testing, as hyperlactatemia reflects both decreased hepatic clearance by a damaged liver and global illness severity and multi-organ failure. Initial reports confirmed their prognostic value, and their inclusion was found to improve specificity, sensitivity and timeliness of the KCC [16]. Other studies have also examined arterial blood lactate in ALF, finding elevated levels to be strongly and independently associated with death or transplantation in both paracetamol and non-paracetamol-induced disease [17-19]. Analysis of the performance of the specific thresholds for arterial lactate introduced into the KCC for paracetamol-induced disease has been less consistent, with one report suggesting that early levels resulted in an increase in sensitivity but at the expense of reduction in specificity [17]. However, two other studies found that lactate measurements alone $12 \mathrm{~h}$ after admission to transplant centers had high predictive accuracy, with performance greater than the KCC alone [18, 19]. Though adopted into United Kingdom ALF wait-listing criteria, meta-analysis of validation studies to date has failed to confirm an improvement in diagnostic test performance through their inclusion [20].

Two further meta-analyses of the performance of the $\mathrm{KCC}$ have further illustrated their potential shortcomings (Fig. 1). Review of studies of the KCC in predicting outcome of non-paracetamol-induced ALF comprising 1105 cases, showed overall sensitivity of $68 \%$ and specificity $82 \%$ [21]. Specificity was highest $(93 \%)$ in patients with high-grade $\mathrm{HE}$ and where the criteria were sequentially determined through the clinical course of illness. Importantly, this analysis also described a fall-off in diagnostic performance in more recent studies, reflecting the increased success of non-transplant approaches to care. A second, larger meta-analysis examining criteria performance in both paracetamol and non-paracetamol etiologies suggested an overall sensitivity of $59 \%$ and specificity of $79 \%$, and with better performance values for prediction of nonsurvival in paracetamol than in non-paracetamol etiologies [22].

Indications are, therefore, of particular aspects where diagnostic performance of selection criteria could be improved beyond existing systems. First, they should consider etiology of illness and reflect the differential in current outcomes of medical care alone. Second, they would perform sequential rather than single time point estimations of prognosis, a process identified on metaanalysis as having additional diagnostic value and reflecting the rapidly changing clinical condition seen particularly in paracetamol and other hyper-acute etiologies and already utilized in other novel prognostic criteria [23]. Recent studies suggest that in these cases waitlist improvement rather than deterioration may now principally confound decision making [9, 11, 12, 15]. Additionally, any new or supplemental criteria should ideally retain the clinical simplicity of existing systems. Two recent attempts to address these issues are discussed below, both of which have utilized the recent availability of web- and app-based computing power to deliver outcome predictions through sophisticated algorithms.

\section{Dynamic outcome predictive model for paracetamol-induced ALF}

Prediction models in hyper-acute disease would be of most benefit if they could be applied sequentially identifying and quantifying both improvement and deterioration [22, 23]. The latest model from the King's Liver Intensive Care Unit is a dynamic outcome prediction model developed and validated for use in patients with paracetamol-induced ALF [24]. It is based on prospective data including analysis of more than 20 daily variables sequentially assessed for 3 days after ICU admission in 912 un-transplanted patients between 2000 and 2012. The variables included in the final models to predict death-included age, hepatic encephalopathy, cardiovascular failure, INR, creatinine and arterial $\mathrm{pH}$ on admission and dynamic variables of changing arterial blood lactate and INR. On validation in independent datasets from four transplant centers, the models showed good discrimination between survivors and non-survivors, improving with the inclusion of changes in INR and Lactate over time (Fig. 2). Innovative in this approach was its access though a dedicated website and the generation of continuous survival estimates rather than a binary survival outcome, with the intention that the model should act as a decision-support tool to support clinical 
a Sensitivity

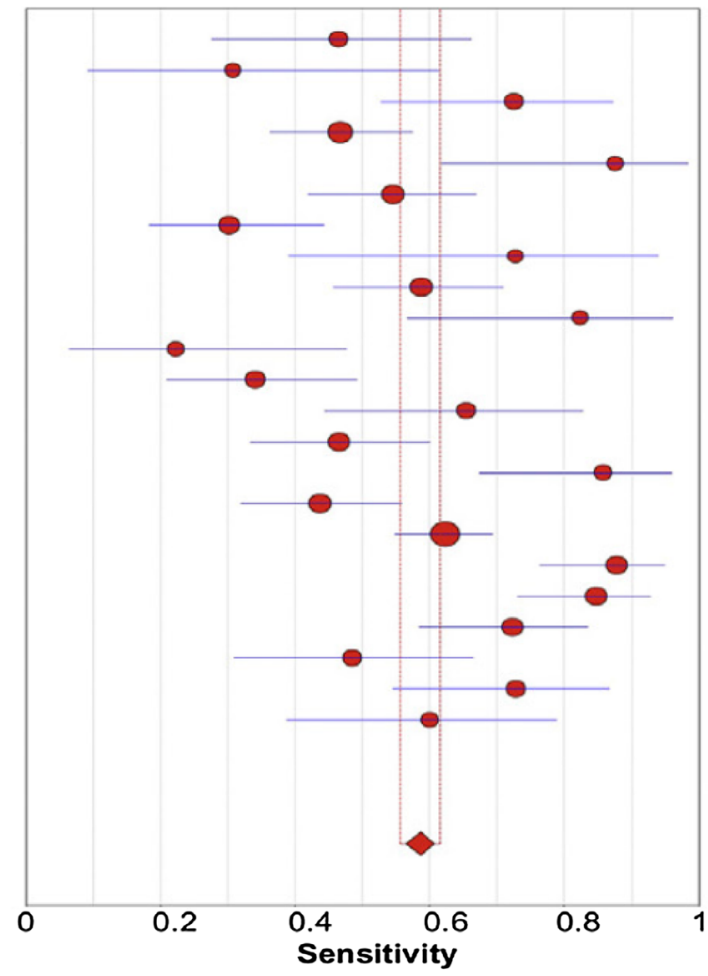

b Specificity

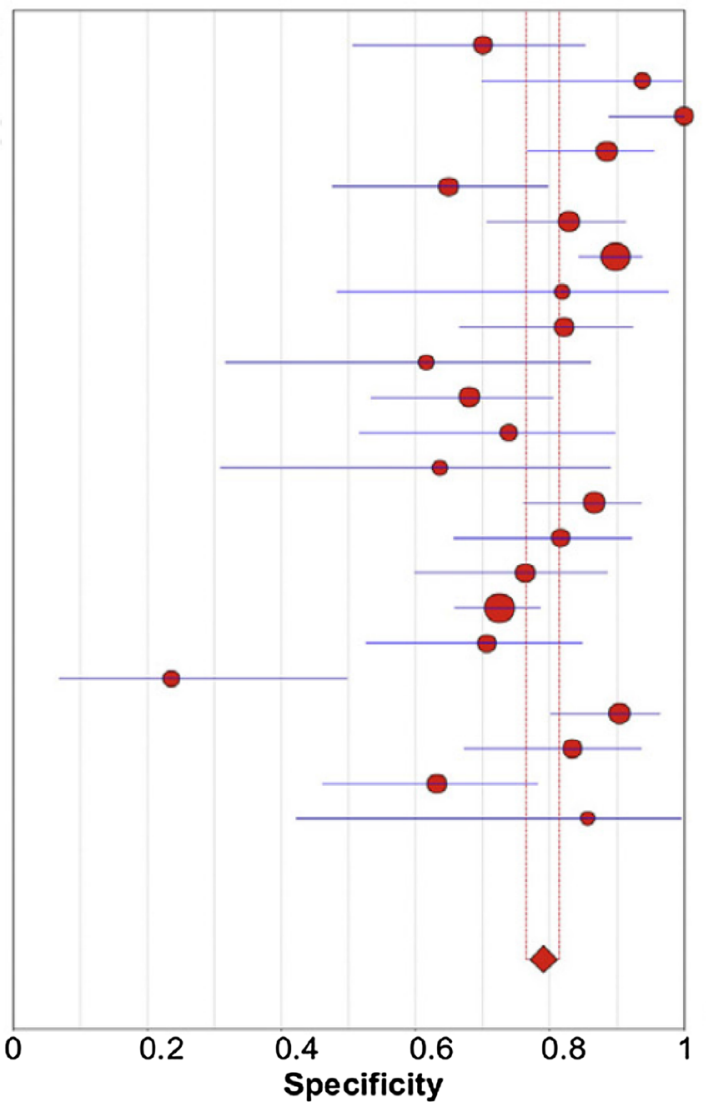

Pelaez-Luna et al 2006 Taylor et al 2006

Zaman et al 2006

Dhiman et al 2007

Katoonizadeh et al 2007

Schmidt \& Larsen 2007

Wei et al 2007

Yantorno et al 2007

Hadem et al 2008

Yamagishi et al 2009

Bechmann et al 2010

Kumar et al 2010

Chen et al 2011

Cholongitas et al 2012

Craig et al 2012

Hadem et al 2012

Kumar et al 2012

Parkash et al 2012

Shaikh et al 2012

Manka et al 2013

Feng et al 2014

Mendizabal et al 2014

Bagchi et al 2015
Sensitivity $(95 \% \mathrm{CI})$

$0.46(0.28-0.66)$

$0.31 \quad(0.09-0.61)$

$0.72(0.53-0.87)$

$0.47 \quad(0.36-0.57)$

$0.88 \quad(0.62-0.98)$

$0.55 \quad(0.42-0.67)$

$0.30 \quad(0.18-0.44)$

$0.73 \quad(0.39-0.94)$

$0.59 \quad(0.46-0.71)$

$0.82(0.57-0.96)$

$0.22(0.06-0.48)$

$0.34 \quad(0.21-0.49)$

$0.65 \quad(0.44-0.83)$

$0.47 \quad(0.33-0.60)$

$0.86(0.67-0.96)$

$0.44 \quad(0.32-0.56)$

$0.62(0.55-0.69)$

$0.88 \quad(0.76-0.95)$

$0.85 \quad(0.73-0.93)$

$0.72(0.58-0.84)$

$0.48 \quad(0.31-0.66)$

$0.73(0.54-0.87)$

$0.60 \quad(0.39-0.79)$

Pooled Sensitivity $=0.59(0.56$ to 0.62$)$

Chi-square $=136.35 ; \mathrm{df}=22(P=.0000)$

Inconsistency (I-square) $=83.9 \%$

Pelaez-Luna et al 2006

Taylor et al 2006

Zaman et al 2006

Dhiman et al 2007

Katoonizadeh et al 2007

Schmidt \& Larsen 2007

Wei et al 2007

Yantorno et al 2007

Hadem et al 2008

Yamagishi et al 2009

Bechmann et al 2010

Kumar et al 2010

Chen et al 2011

Cholongitas et al 2012

Craig et al 2012

Hadem et al 2012

Kumar et al 2012

Parkash et al 2012

Shaikh et al 2012

Manka et al 2013

Feng et al 2014

Mendizabal et al 2014

Bagchi et al 2015
Specificity $(95 \% \mathrm{CI})$

0.70

$1.00 \quad(0.89-1.00)$

$0.88 \quad(0.77-0.96)$

$0.65 \quad(0.47-0.80)$

$0.83 \quad(0.71-0.91)$

$0.90 \quad(0.84-0.94)$

$0.82 \quad(0.48-0.98)$

$0.82 \quad(0.66-0.92)$

$0.62 \quad(0.32-0.86)$

$0.68 \quad(0.53-0.80)$

$0.74 \quad(0.52-0.90)$

$0.64 \quad(0.31-0.89)$

$0.87 \quad(0.76-0.94)$

$0.82 \quad(0.66-0.92)$

$0.76 \quad(0.60-0.89)$

$0.73 \quad(0.66-0.79)$

$0.71 \quad(0.53-0.85)$

$0.24 \quad(0.07-0.50)$

$0.90 \quad(0.80-0.96)$

$0.83 \quad(0.67-0.94)$

$0.63 \quad(0.46-0.78)$

$0.86(0.42-1.00)$

Pooled Specificity $=0.79(0.77$ to 0.81$)$

Chi-square $=92.38 ; \mathrm{df}=22(P=.0000)$

Inconsistency (I-square) $=76.2 \%$

Fig. 1 Pooled (a) Sensitivity and (b) Specificity of Kings College Criteria (Source Ref. [20]) 


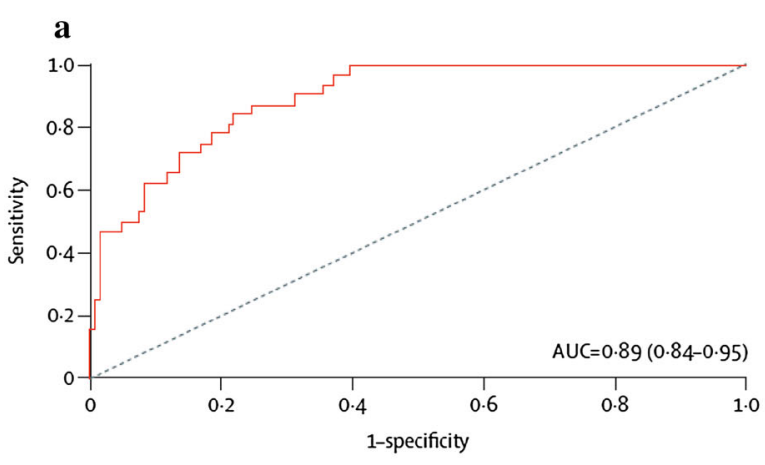

c

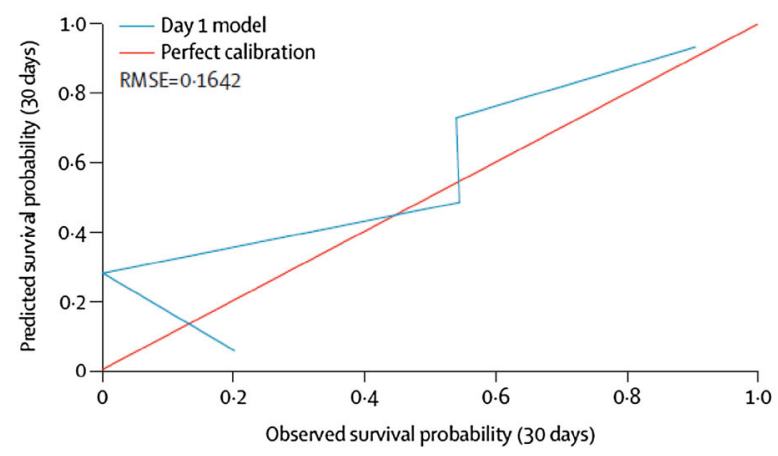

Fig. 2 Discrimination and calibration of dynamic prediction model on day 1 and day 2 of admission in 150 patients with paracetamolinduced ALF. a Area under receiver operating characteristic curve

judgement rather than a sole arbiter as to proceeding with transplantation.

\section{Model of acute liver failure study group (ALFSG)}

Elements of this approach were also adopted in the recent analysis by the ALFSG of 1974 subjects enrolled prospectively from 28 academic centers across North America between 1998 and 2013, and managed with and without transplantation [25]. The aim was to devise a mathematical model for all etiologies of ALF to predict transplant-free survival at 21 days, rather than mortality as adopted in other models-making comparison with other studies adopting more standard approaches more complex. Clinical features and laboratory values were collected at study enrolment and recorded serially up to 7 days. Variables of prognostic value adopted in the predictive model included admission coma grade, etiology and vasopressor requirement, and admission bilirubin and INR values. Arterial blood lactate was not explored. In this analyses of both paracetamol and non-paracetamol cases, sequential values of the INR did not add to prediction over that on admission. Using AUROC analysis, test discrimination appeared superior to the KCC and MELD scores (Fig. 3) b

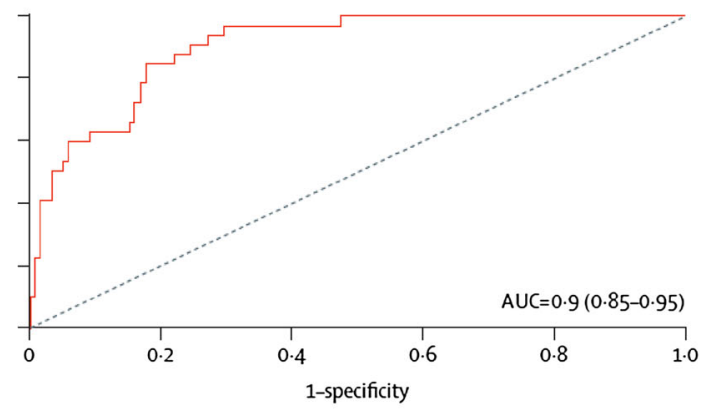

d

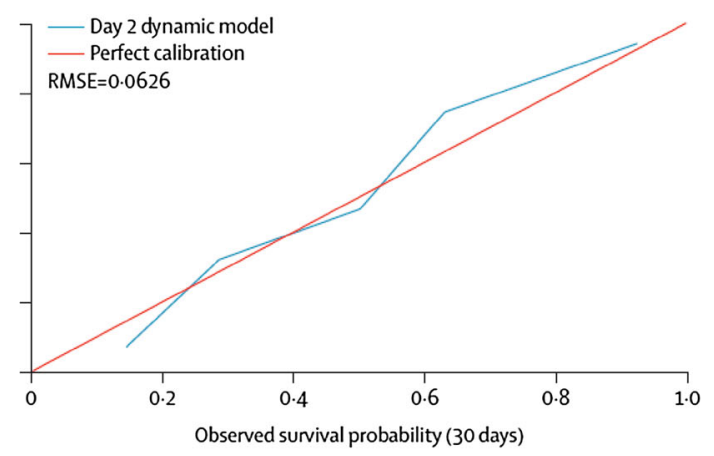

(AUROC) for day 1 model, b AUROC for day 2 model, c Calibration curve for day 1 model, $\mathbf{d}$ Calibration curve for day 2 model (Source Ref. [21])

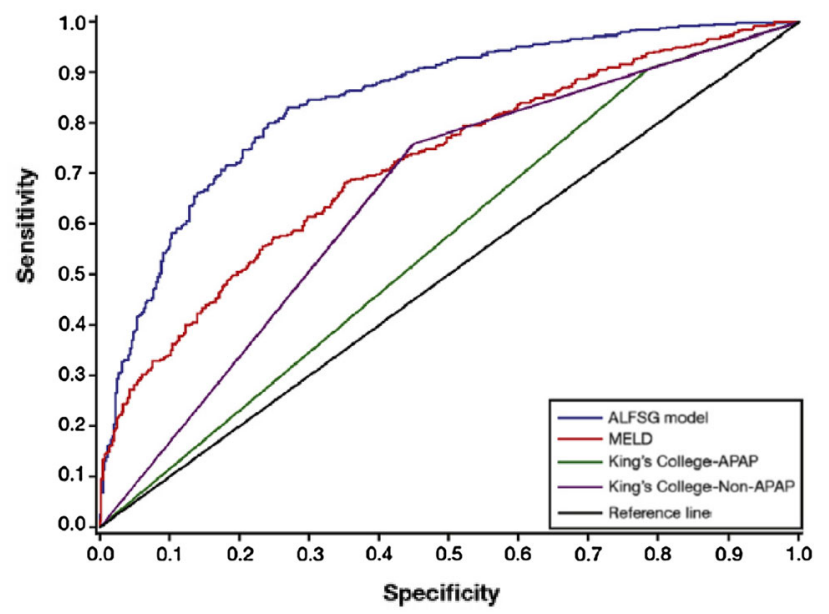

Fig. 3 Comparison of the ALFSG model with the Kings College Criteria and MELD score in predicting survival. ALFSG acute liver failure study group, MELD model for end stage liver disease APAP acetaminophen (paracetamol). AUROC: ALFSG: 0.843, MELD: 0.717, KCC APAP 0.560, KCC non-APAP 0.655 (Source Ref. [22])

with the model correctly predicting outcome of illness in $66.3 \%$ of subjects. Its performance was best in patients with unfavorable etiologies and high-grade encephalopathy and more limited in those with more favorable etiologies and high-grade encephalopathy. 
Though sharing similarities in their use of web-based predictive algorithms, key differences exist between these two predictive systems. One is based on the prediction of mortality and the other survival, with resultant differences in test performance. Further, they are targeted at different patient groups-with the USALF criteria taking a broad approach to all etiologies of ALF, whilst the Dynamic Model is focused upon liver injury from paracetamol where the USALF performs least well. It may be that application of these and other models are complimentary and decision making is based upon the results generated from several prognostic systems [23-25].

\section{Other systems evaluated}

A variety of other scoring systems and supplemental markers have been proposed to identify candidates for liver transplantation, though in general their performance has been evaluated in patient cohorts of limited size (Table 2). Scores used in this way include non-liver specific ones such as SOFA and APACHE II that are widely used to quantify severity of multi-organ failure in other forms of critical illness [17, 26, 27]. Reports suggest similar sensitivity and specificity to the King's criteria in predicting death and they can be applied sequentially. However, though they may identify those patients at risk of death, the multi-organ failure they quantify may not be corrected by transplantation, if for example, it results for other ALFassociated complications such as severe sepsis or pancreatitis. Consequently, they are not in widespread use to select candidates for transplant.

The MELD score is more focused on severity of liver injury and has been assessed more widely in ALF and subjected to meta-analysis [22, 28, 29]. In its unmodified form, it shows most promise in non-paracetamol etiologies, with diagnostic performance close to that of the KCC [22]. It has also been combined with circulating blood levels of cytokeratin K18 (CK18) a cell death-associated marker measured using the M30 assay which principally reflects apoptotic cell death [30]. Also, this measurement in place of bilirubin to give a modified MELD Score showed superior sensitivity and specificity to the standard MELD Score and the King's criteria in predicting outcome of ALF [31].

Other studies have also reported prognostic models combining standard clinical variables with non-standard analytes. By example, the ALFSG index predictive model combined Coma-grade, INR, bilirubin and phosphorus levels with that of blood levels of M30 CK18 [32]. In a 250 -strong validation cohort, discrimination between survivors and non-survivors was greater than with the KCC or MELD, though later external validation in a smaller

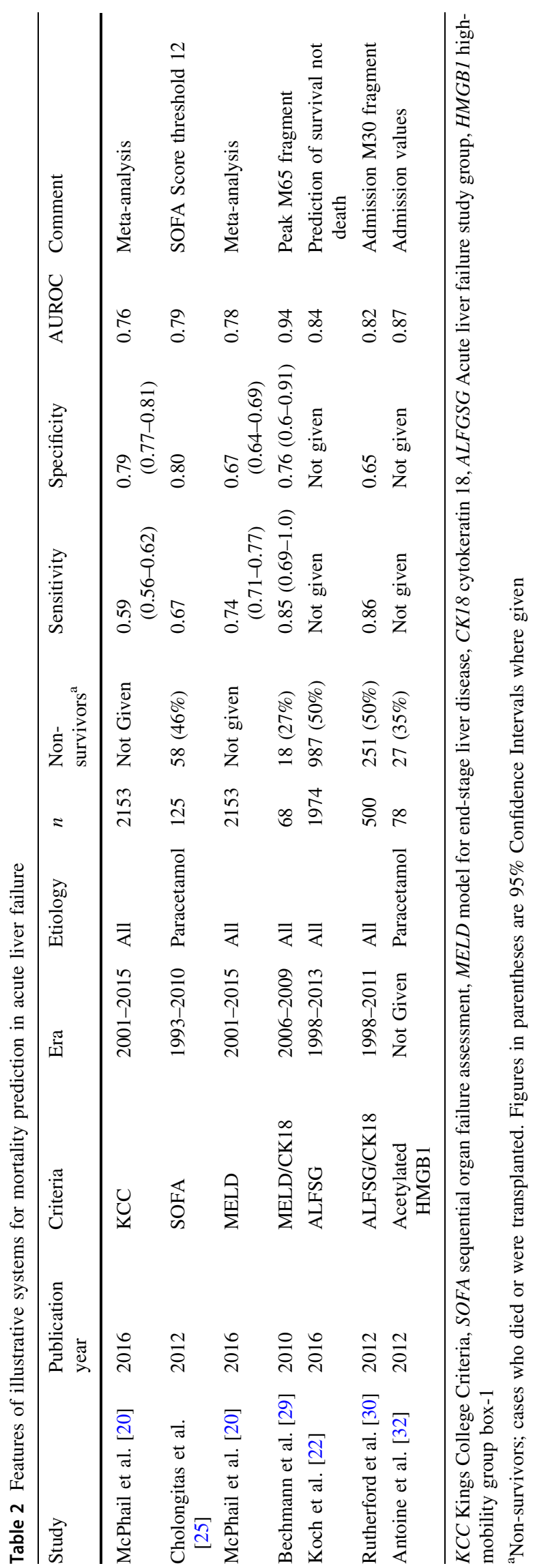


independent cohort showed no advantage over SOFA or APACHE scoring [32, 33].

However, the primary mechanism of liver cell injury may vary by etiology and necrosis rather than apoptosis may predominate. Antoine and colleagues assessed circulating levels of a panel of cell death markers in 78 patients with paracetamol-induced ALF [34]. They found that best prediction of non-survival was with acetylated HMGBI-a biomarker of hepatic necrosis and cellular immunological activation, and to lesser degree with biomarkers of apoptosis including molecular forms of CK18. To date, validation studies have not indicated prognostic advantage above the standard KCC in predicting survival [35]. Cell death biomarkers do appear to be exquisitely sensitive in predicting clinically significant liver injury very early after paracetamol overdose but If they are to be adopted as adjunctive measures to select liver transplant candidates with ALF, key practical issues will need to be overcome $[36,37]$. Techniques for their rapid determination will need to be widely available, and it is likely that a panel of biomarkers will need to be assessed to cover etiologyspecific differences in the mechanisms of liver cell injury and death.

Other simpler clinical measures have also demonstrated potential for use as prognostic markers: platelet count has been shown to be closely linked to outcome. In a recent study from the USALFSG, the evolution of thrombocytopenia was closely associated with development of multiorgan failure and a poor outcome in ALF and linked to the development of a systemic inflammatory response [38] (Fig. 4a). Liver volume may be easily determined using analysis of CT images and reflect the balance between the parallel processes of liver collapse with cell death and increase with regeneration. Recent studies suggest that in some non-paracetamol etiologies, loss of liver volume in adults to less than $1000 \mathrm{~cm}^{3}$ may indicate irreversible damage and serve as an early indicator of poor prognosis, often in advance of the development of encephalopathy (Fig. 4b) [39-41].

Other studies have also sought to identify at a very early stage those patients with acute liver injury (ALI) in the absence of encephalopathy which will later progress to a poor outcome of death or transplantation. In a study of US patients hospitalized with ALI, progression to a poor outcome was more common in non-paracetamol etiologies and with higher levels of INR and bilirubin, and a prolonged duration of illness [42].

Finally, it is important to emphasize that prognostic models should be only part of the overall functional evaluation of the very sick patient with ALF and an experienced multi-disciplinary team in an intensive care setting is required for correct interpretation. Rather than providing an
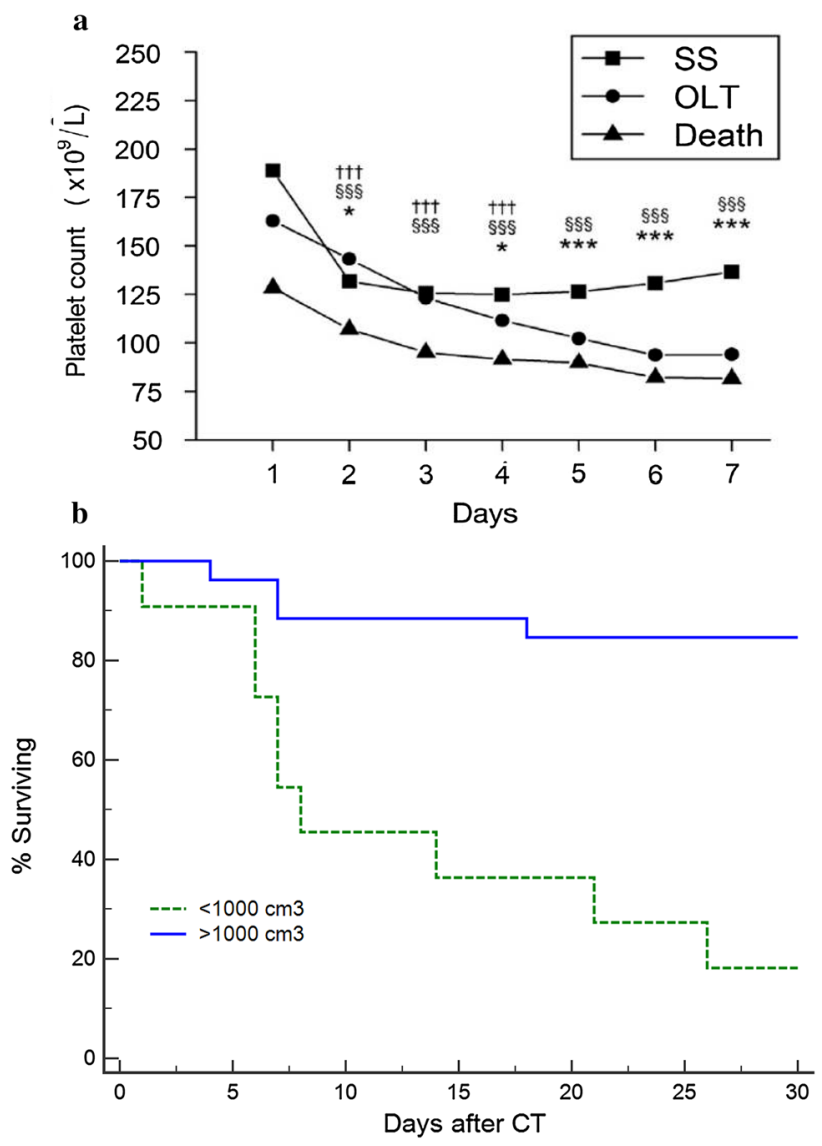

Fig. 4 a Platelet Counts on days 1-7 after admission in 1598 patients with acute liver failure according to outcome at 21 days. SS spontaneous survivor, $O L T$ orthotropic liver transplant. Three symbols, $p<0.001$; two symbols, $p<0.01$; one symbol, $p<0.05$. *SS vs LT, ${ }^{\S}$ SS vs death, and ${ }^{\dagger}$ LT vs death. SS, spontaneous survival. b Survival According to CT-derived liver volume on admission in 37 non-transplanted patients with non-acetaminophen etiologies. $<1000 \mathrm{~cm}^{3} \quad n=11, \geq 1000 \mathrm{~cm}^{3} \quad n=26, \quad p<0.001 \quad$ log-rank (Source Ref. [36, 38])

absolute arbiter, these models should support decision making and the multifactorial team assessment.

\section{Current results of liver transplantation}

Life-saving though it was soon seen to be after its introduction, overall results not surprisingly for these very sick patients were significantly lower than for elective transplants carried out for those with chronic diseases. Several series have now demonstrated a progressive incremental improvement in survival over time, with patient survival now approaching that of elective surgery. Recent reports for the US and UK are of 1-year patient survival rates of 85-90\% [43]. These results reflect advances in peri- and intra-operative care and the interplay of several factors: the earlier and more accurate prediction of need for transplant 
and identification those patients who are too sick for surgery, and a better understanding of the interplay between graft and recipient factors in determining patient survival. Analysis of the European experience of 4903 transplant recipients between 1998 and 2009 found death after surgery to relate to both with independent association shown with male recipients (adjusted OR 1.25), recipient $>50$ years of age (1.26), incompatible ABO matching (1.93), donors $>60$ years (1.21), and reduced size graft (1.54). Recipients $>50$ years, combined with donors $>60$ years had a $57 \%$ mortality/graft loss within the first year after transplant [44]. Matching of graft to recipient is key to ensuring optimal outcome [13]. Case series also now confirms the practicality and excellent outcomes obtained for selected patients transplanted using living donors, and from auxiliary liver grafting in etiologies where native liver regenerative potential exists-though globally, these techniques are applied to only a small proportion of recipients $[45,46]$.

Patient survival after transplantation for ALF follows a characteristic pattern of increased mortality early after transplant, but in patients who survive this phase, subsequent survival parallels closely with that seen in elective transplantation [44]. In an early series, the high prevalence of psychiatric comorbidity and addiction issues seen in recipients of transplantation for paracetamol-induced ALF was reflected in an increased risk of death due to suicide, trauma or non-adherence to immunosuppression with over half the deaths occurring within 12 months of transplantation [44]. Later series have not demonstrated such an increase in mortality, but it is clear that paracetamol recipients show increased levels of psychotic comorbidity which may be reflected in poor compliance with medication and follow-up [47]. In these patients, assessment of severity of psychiatric illness is a key element of the selection of potential recipients and in those transplanted follow-up must include close ongoing psychiatric monitoring and support.

\section{New novel non-transplant options}

A number of novel non-transplant interventions have potential to serve as alternatives to transplantation. A central premise to their use is that the injured liver retains regenerative ability and that this may be augmented by these interventions. A better practical understanding of the processes influencing both liver injury and regeneration is likely to be of clinical importance here, exemplified by the major clinical and laboratory differences between paracetamol hepatotoxity from single time point or staggered overdoses - and the markedly worse outcomes seen in the latter group [48]. The potentially important influence of pre-existing sub-clinical non-cirrhotic liver disease upon liver regeneration is currently poorly characterized, for example, from chronic alcohol use or non-alcoholic steatohepatitis.

In cases where the liver injury has progressed 'beyond the point of no return', novel medical therapies are unlikely to be of benefit. It would thus be of no surprise that most efficacy would be seen either in treatment at an early stage of disease and/or in etiologies with greatest regenerative capacity. By example, the FULMAR trial of the MARS extra-corporeal device in patients with ALF failed to show a survival improvement in the overall study cohort but there were indications of potential benefit in patients with paracetamol-related disease [49].

Such caveats may also apply to the use of therapeutic plasma exchange (TPE). Through mechanisms thought to include removal of deleterious inhibitors of hepatic regeneration and a complex pattern of immunomodulation, use of this therapy has recently been demonstrated to deliver a survival benefit in non-transplanted patients with ALF. In a multi-center randomized controlled trial on mixed etiologies of disease, three 5-1 TPE sessions significantly improved survival above that seen with standard medical care, although the survival benefit was inferior to that seen with transplantation (Fig. 5) [50]. There is ongoing debate about the place of this therapy in patients with ALF, given that its use normalizes measures of coagulation disturbance and thus precludes their use in prognostic evaluation for later transplantation. Our practice is in accordance with recent EASL guidance, with its early use in patients with ALF with an expected poor prognosis without transplantation but who have clear medical or psychiatric contra-indications to surgery [51]. In addition, the use of TPE is associated with significant improvements in cardiovascular status and we also treat patients who are waitlisted for liver transplantation but with worsening

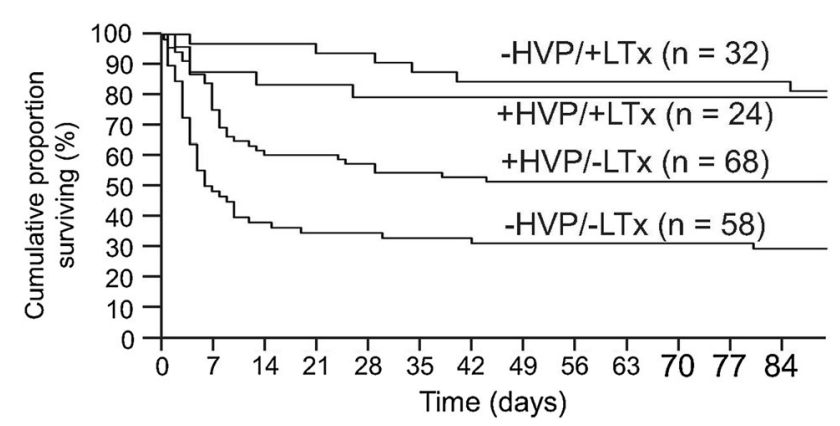

Fig. 5 Survival in 182 patients with ALF according to treatment with liver transplantation and high-volume plasma exchange. Two groups receiving SMT (standard medical treated group) with and without emergency transplantation (HVP + LTx vs +HVPLTx) and the two group receiving SMT with and without emergency transplantation (HVPLTx vs. +HVPLTx) (log rank: $p=0.0058)$ and Cox proportional hazard: LTx: $p<0.0001$; HVP: $p=0.0076)$ (Source Ref. [48]) 
cardiovascular failure and vasopressor requirement while awaiting a graft [50].

Other forms of cellular and immune-modulatory therapy show promise as future treatments for ALF. Whilst hepatocyte transplantation retains considerable theoretical attractions, it presents considerable logistic challenges and a requirement for immunosuppression, and its benefit is yet to be established in adult patients with ALF [52, 53]. Other interventions in patients with acute-on-chronic liver failure (ACLF) show promise but are yet to be tested in ALF. Understanding of the pathophysiologic basis of ACLF is rapidly increasing and the insights gained, particularly in relation to modulation of regeneration and immunological function, may well be transportable to ALF. In a randomized controlled single-blind trial in 110 patients with Hepatitis B-related ACLF of whom less than half were thought to be cirrhotic, treatment with allogenic mesenchymal stem cells improved survival-[54]. Similarly, in a single-center double-blind trial in 55 patients with decompensated cirrhosis, treatment with granulocyte colony stimulating factor and erythropoietin was associated with improved survival in association with indications of improved hepatic regeneration through mobilization of progenitor cells [55]. These results are yet to be replicated or tested in patients with ALF but demonstrate mechanistic approaches likely to be assessed in the near future. As ever, the rarity and severity of ALF represent major limiting factors for performing randomized controlled trials of these or other agents and true advances in therapeutic intervention are likely to result only from international collaborative studies.

\section{Compliance with ethical standards}

Conflict of interest Professor Roger Williams and Professor William Bernal have no potential conflict of interest.

Open Access This article is distributed under the terms of the Creative Commons Attribution 4.0 International License (http://creative commons.org/licenses/by/4.0/), which permits unrestricted use, distribution, and reproduction in any medium, provided you give appropriate credit to the original author(s) and the source, provide a link to the Creative Commons license, and indicate if changes were made.

\section{References}

1. Gazzard BG, Widdop B, Davis M, Hughes RD, Goulding R, Williams R. Early prediction of the outcome of a paracetamol overdose based on an analysis of 163 patients. Postgrad Med J 1977;53(619):243-247.

2. O'Grady JG, Alexander GJ, Hayllar KM, Williams R. Early indicators of prognosis in fulminant hepatic failure. Gastroenterology 1989;97(2):439-445.
3. O'Grady JG, Schalm SW, Williams R. Acute liver failure: redefining the syndromes. Lancet 1993;342(8866):273-275.

4. Bernal W, Hyyrylainen A, Gera A, et al. Lessons from look-back in acute liver failure? A single centre experience of 3300 patients. J Hepatol 2013;59(1):74-80.

5. Slack A, Auzinger G, Willars C, et al. Ammonia clearance with haemofiltration in adults with liver disease. Liver Int 2014;34(1):42-48.

6. Cardoso FS, Gottfried M, Tujios S, Olson JC, Karvellas CJ. Group USALFS. Continuous renal replacement therapy is associated with reduced serum ammonia levels and mortality in acute liver failure. Hepatology 2017;31:31.

7. O'Grady J. Timing and benefit of liver transplantation in acute liver failure. J Hepatol 2014;60(3):663-670.

8. Donnelly MC, Davidson JS, Martin K, Baird A, Hayes PC, Simpson KJ. Acute liver failure in Scotland: changes in aetiology and outcomes over time (the Scottish Look-Back Study). Aliment Pharmacol Ther 2017;45(6):833-843.

9. Reuben A, Tillman H, Fontana R, et al. Outcomes in Adults With Acute Liver Failure Between 1998 and 2013: an observational cohort study. Ann Intern Med 2016;164(11):724-732.

10. Oketani M, Ido A, Nakayama N, et al. Etiology and prognosis of fulminant hepatitis and late-onset hepatic failure in Japan: summary of the annual nationwide survey between 2004 and 2009 . Hepatol Res 2013;43(2):97-105.

11. Ichai $P$, Legeai $C$, Francoz $C$, et al. Patients with acute liver failure listed for superurgent liver transplantation in France: reevaluation of the Clichy-Villejuif criteria. Liver Transpl 2015;21(4):512-523.

12. Simpson KJ, Bates CM, Henderson NC, et al. The utilization of liver transplantation in the management of acute liver failure: comparison between acetaminophen and non-acetaminophen etiologies. Liver Transpl 2009;15(6):600-609.

13. Bernal W, Cross TJS, Auzinger G, et al. Outcome after waitlisting for emergency liver transplantation in acute liver failure: a single centre experience. J Hepatol 2009;50(2):306-313.

14. Brandsaeter B, Hockerstedt K, Friman S, et al. Fulminant hepatic failure: outcome after listing for highly urgent liver transplantation-12 years experience in the nordic countries. Liver Transpl 2002;8(11):1055-1062.

15. Reddy KR, Ellerbe C, Schilsky M, et al. Determinants of outcome among patients with acute liver failure listed for liver transplantation in the United States. Liver Transpl 2016;22(4):505-515.

16. Bernal W, Donaldson N, Wyncoll D, Wendon J. Blood lactate as an early predictor of outcome in paracetamol-induced acute liver failure: a cohort study. Lancet 2002;359(9306):558-563.

17. Schmidt LE, Larsen FS. Prognostic implications of hyperlactatemia, multiple organ failure, and systemic inflammatory response syndrome in patients with acetaminophen-induced acute liver failure. Crit Care Med 2006;34(2):337-343.

18. Macquillan GC, Seyam MS, Nightingale P, Neuberger JM, Murphy N. Blood lactate but not serum phosphate levels can predict patient outcome in fulminant hepatic failure. Liver Transpl 2005;11(9):1073-1079.

19. Cholongitas E, O’Beirne J, Betrossian A, et al. Prognostic impact of lactate in acute liver failure. Liver Transpl 2008;14(1):121-122 (author reply 3).

20. Craig DGN, Ford AC, Hayes PC, Simpson KJ. Systematic review: prognostic tests of paracetamol-induced acute liver failure. Aliment Pharmacol Ther 2010;31(10):1064-1076.

21. McPhail MJW, Wendon JA, Bernal W. Meta-analysis of performance of Kings's College Hospital Criteria in prediction of outcome in non-paracetamol-induced acute liver failure. J Hepatol 2010;53(3):492-499. 
22. McPhail MJ, Farne H, Senvar N, Wendon JA, Bernal W. Ability of King's College Criteria and model for end-stage liver disease scores to predict mortality of patients with acute liver failure: a meta-analysis. Clin Gastroenterol Hepatol 2016;14(4):516-525.e5 (quiz e43-e45).

23. Kumar R, Shalimar Sharma H, et al. Prospective derivation and validation of early dynamic model for predicting outcome in patients with acute liver failure. Gut 2012;61(7):1068-1075.

24. Bernal W, Wang Y, Maggs J, et al. Development and validation of a dynamic outcome prediction model for paracetamol-induced acute liver failure: a cohort study. Lancet Gastroenterol Hepatol 2016;1(3):217-225.

25. Koch DG, Tillman H, Durkalski V, Lee WM, Reuben A. Development of a model to predict transplant-free survival of patients with acute liver failure. Clin Gastroenterol Hepatol 2016;14(8):1199-1206.e2.

26. Craig DG, Zafar S, Reid TW, et al. The sequential organ failure assessment (SOFA) score is an effective triage marker following staggered paracetamol (acetaminophen) overdose. Aliment Pharmacol Ther 2012;35(12):1408-1415.

27. Cholongitas E, Theocharidou E, Vasianopoulou P, et al. Comparison of the sequential organ failure assessment score with the King's College Hospital criteria and the model for end-stage liver disease score for the prognosis of acetaminophen-induced acute liver failure. Liver Transpl 2012;18(4):405-412.

28. Schmidt L, Larsen F. MELD score as a predictor of liver failure and death in patients with acetaminophen-induced liver injury. Hepatology 2007;45:789-796.

29. Craig DGN, Reid TWDJ, Wright EC, et al. The sequential organ failure assessment (SOFA) score is prognostically superior to the model for end-stage liver disease (MELD) and MELD variants following paracetamol (acetaminophen) overdose. Aliment Pharmacol Ther 2012;35(6):705-713.

30. Eguchi A, Wree A, Feldstein AE. Biomarkers of liver cell death. J Hepatol 2014;60(5):1063-1074.

31. Bechmann LP, Jochum C, Kocabayoglu P, et al. Cytokeratin 18-based modification of the MELD score improves prediction of spontaneous survival after acute liver injury. J Hepatol 2010;53(4):639-647.

32. Rutherford A, King LY, Hynan LS, et al. Development of an accurate index for predicting outcomes of patients with acute liver failure. Gastroenterology 2012;143(5):1237-1243.

33. Craig DG, Simpson KJ. Accuracy of the ALFSG index as a triage marker in acute liver failure. Gastroenterology 2013;144(1):e25.

34. Antoine DJ, Jenkins RE, Dear JW, et al. Molecular forms of HMGB1 and keratin-18 as mechanistic biomarkers for mode of cell death and prognosis during clinical acetaminophen hepatotoxicity. J Hepatol 2012;56(5):1070-1079.

35. Craig DGN, Lee P, Pryde EA, Masterton GS, Hayes PC, Simpson KJ. Circulating apoptotic and necrotic cell death markers in patients with acute liver injury. Liver Int 2011;31(8):1127-1136.

36. Dear JW, Clarke JI, Francis B, et al. Risk stratification after paracetamol overdose using mechanistic biomarkers: results from two prospective cohort studies. Lancet Gastroenterol Hepatol 2018;3(2):104-113.

37. Antoine DJ, Dear JW, Lewis PS, et al. Mechanistic biomarkers provide early and sensitive detection of acetaminophen-induced acute liver injury at first presentation to hospital. Hepatology 2013;58(2):777-787.

38. Stravitz RT, Ellerbe C, Durkalski V, et al. Thrombocytopenia Is associated with multi-organ system failure in patients with acute liver failure. Clin Gastroenterol Hepatol 2016;14(4):613-620.

39. Shakil AO, Jones BC, Lee RG, Federle MP, Fung JJ, Rakela J. Prognostic value of abdominal CT scanning and hepatic histopathology in patients with acute liver failure. Dig Dis Sci 2000;45(2):334-339.
40. Zabron A, Quaglia A, Peddu P, et al. Clinical and prognostic associations of liver volume determined by computed tomography in acute liver failure. Liver Int 2018 (In Press).

41. Yamagishi Y, Saito H, Tada S, et al. Value of computed tomography-derived estimated liver volume/standard liver volume ratio for predicting the prognosis of adult fulminant hepatic failure in Japan. J Gastroenterol Hepatol 2005;20(12):1843-1849.

42. Koch DG, Speiser JL, Durkalski V, et al. The natural history of severe acute liver injury. Am J Gastroenterol 2017;112(9):1389-1396.

43. Blood and Transplant N. ANNUAL REPORT ON LIVER TRANSPLANTATION. 2017. https://nhsbtdbe.blob.core.win dows.net/umbraco-assets-corp/5007/annual_liver_transplantation_ report_2017.pdf Accessed 19 Feb 2018.

44. Germani G, Theocharidou E, Adam R, et al. Liver transplantation for acute liver failure in Europe: outcomes over 20 years from the ELTR database. J Hepatol 2012;57(2):288-296.

45. Yamashiki N, Sugawara Y, Tamura S, et al. Outcomes after living donor liver transplantation for acute liver failure in Japan: results of a nationwide survey. Liver Transpl 2012;18(9):1069-1077.

46. Goldaracena N, Spetzler VN, Marquez M, et al. Live donor liver transplantation: a valid alternative for critically ill patients suffering from acute liver failure. Am J Transplant 2015;15(6):1591-1597.

47. Karvellas CJ, Safinia N, Auzinger G, et al. Medical and psychiatric outcomes for patients transplanted for acetaminophen-induced acute liver failure: a case-control study. Liver Int 2010;30(6):826-833.

48. Craig DGN, Bates CM, Davidson JS, Martin KG, Hayes PC, Simpson KJ. Staggered overdose pattern and delay to hospital presentation are associated with adverse outcomes following paracetamol-induced hepatotoxicity. $\mathrm{Br} \quad \mathrm{J}$ Clin Pharmacol 2012;73(2):285-294.

49. Saliba F, Camus C, Durand F, et al. Albumin dialysis with a noncell artificial liver support device in patients with acute liver failure: a randomized, controlled trial. Ann Intern Med 2013;159(8):522-531.

50. Larsen FS, Schmidt LE, Bernsmeier C, et al. High-volume plasma exchange in patients with acute liver failure: an open randomised controlled trial. J Hepatol 2016;64(1):69-78.

51. European Association for the Study of the Liver. Electronic address eee, Clinical practice guidelines $\mathrm{p}$, Wendon $\mathrm{J}$, et al. EASL Clinical Practical Guidelines on the management of acute (fulminant) liver failure. J Hepatol 2017;66(5):1047-1081.

52. Iansante V, Mitry RR, Filippi C, Fitzpatrick E, Dhawan A. Human hepatocyte transplantation for liver disease: current status and future perspectives. Pediatr Res 2017;06:06.

53. Ibars EP, Cortes M, Tolosa L, et al. Hepatocyte transplantation program: lessons learned and future strategies. World J Gastroenterol 2016;22(2):874-886.

54. Lin BL, Chen JF, Qiu WH, et al. Allogeneic bone marrowderived mesenchymal stromal cells for hepatitis B virus-related acute-on-chronic liver failure: a randomized controlled trial. Hepatology 2017;66(1):209-219.

55. Kedarisetty CK, Anand L, Bhardwaj A, et al. Combination of granulocyte colony-stimulating factor and erythropoietin improves outcomes of patients with decompensated cirrhosis. Gastroenterology 2015;148(7):1362-1370 (e7).

56. Bernuau J, Rueff B, Benhamou JP. Fulminant and subfulminant liver failure: definitions and causes. Semin Liver Dis 1986;6(2):97-106.

57. Mochida S, Nakayama N, Matsui A, Nagoshi S, Fujiwara K. Reevaluation of the Guideline published by the Acute Liver Failure Study Group of Japan in 1996 to determine the indications of liver transplantation in patients with fulminant hepatitis. Hepatol Res 2008;38(10):970-979. 